\title{
Review
}

\section{Autophagy and multivesicular bodies: two closely related partners}

\author{
$\mathrm{CM}$ Fader $^{1}$ and MI Colombo ${ }^{*, 1}$
}

In the majority of cell types, multivesicular bodies (MVBs) are a special kind of late endosomes, crucial intermediates in the internalization of nutrients, ligands and receptors through the endolysosomal system. ESCRT-0, I, II and III (endosomal sorting complex required for transport) are involved in the sorting of proteins into MVBs, generating the intraluminal vesicles. Autophagy is a lysosomal degradation pathway for cytoplasmic components such as proteins and organelles. The autophagosome, a well-characterized structure of the autophagy pathway, can fuse with endocytic structures such as MVBs to generate the amphisome. Finally, the amphisome fuses with the lysosome to degrade the material wrapped inside. Currently, clear evidence suggests that efficient autophagic degradation requires functional MVBs. This review highlights the most recent advances in our understanding of the molecular machinery that participates in MVB biogenesis and regulates the interplay between autophagy and this organelle.

Cell Death and Differentiation (2009) 16, 70-78; doi:10.1038/cdd.2008.168; published online 14 November 2008

The endocytic pathway is a dynamic system that functions in cellular housekeeping by internalization, transport, sorting and degradation of macromolecules. ${ }^{1}$ Some receptors, such as epidermal growth factor receptor (EGFR), are removed from the plasma membrane by incorporation into endocytic structures, which can fuse with an early endosome (also known as a sorting endosome). Next, the EGFR is selectively sorted by budding and subsequent pinching off of the endosomal membrane into the luminal space, leading to the formation of multivesicular bodies (MVBs). MVBs, a morphologically distinctive late endosome, accumulate internally small membrane vesicles $(60-80 \mathrm{~nm})$ containing the cargo protein. Finally, MVBs can fuse with the lysosome to degrade the proteins sequestered by acidic hydrolases (Figure 1). Other receptors, such as the transferrin receptor (TfR) or lowdensity lipoprotein (LDL) receptor (after releasing their iron or LDL, respectively), recycle back to the plasma membrane through recycling endosomes. However, in specialized cell types such as reticulocytes, precursors of the mature red blood cell, TfR is accumulated in the internal vesicles of MVBs and released into the extracellular medium by fusion of MVBs with the limiting plasma membrane (i.e. exosomes) ${ }^{2-7}$ (Figure 1). Each cargo protein, destined for either recycling or exocytosis (TfR) or degradation (EGFR), transits through the sorting (early) endosome, where they are selected and targeted to each specific pathway.

Maturation of a red blood cell requires not only getting rid of certain plasma membrane proteins such as the TfR as indicated above but also a series of ultrastructural changes, which include discarding the nucleus and organelles. ${ }^{8}$ Autophagy, a lysosomal degradation pathway, is the main physiological pathway for the degradation of intracellular macromolecules in eukaryotic cells,, 10 playing a specific function in the turnover of organelles, long-lived proteins, RNA (for a review, see Tanida et al. ${ }^{11}$ ). This process is initiated when a cup-shaped structure called phagophore or isolation membrane, which probably originates from specialized regions of the ER, engulfs part of the cytoplasm or organelles, forming a closed double-membraned vacuole termed autophagosome. This vacuolar structure, with $300-900 \mathrm{~nm}$ of diameter, then undergo a maturation process including fusion events with early and late endosomal vesicles, such as the MVBs, forming the amphisome, a hybrid organelle. Subsequently, the amphisome fuses with lysosomes (forming an autolysosome) to degrade the incorporated material. ${ }^{12-16}$ Finally, the digested materials are transported back to the cytoplasm and reused.

\footnotetext{
${ }^{1}$ Laboratorio de Biología Celular y Molecular, Instituto de Histología y Embriología (IHEM)-CONICET, Facultad de Ciencias Médicas, Universidad Nacional de Cuyo, Casilla de Correo 56, Centro Universitario, Parque General San Martín, 5500 Mendoza, Argentina

*Corresponding author: MI Colombo, Laboratorio de Biología Celular y Molecular, Instituto de Histología y Embriología (IHEM)-CONICET, Facultad de Ciencias Médicas, Universidad Nacional de Cuyo, Casilla de Correo 56, Centro Universitario, Parque General San Martín, Mendoza 5500, Argentina.

Tel: + 54261449 4143; Fax: + 54261449 4117; E-mail: mcolombo@fcm.uncu.edu.ar

Keywords: multivesicular bodies; ESCRT machinery; autophagy; LC3; exosomes; K562 cells

Abbreviations: MVB, multivesicular body; ESCRT, endosomal sorting complex required for transport; ConA, concanavalin A; WGA, wheat germen agglutinin; Hrs, hepatocyte growth factor-regulated tyrosine kinase substrate; Vps, vacuolar sorting protein; CHMP, charged multivesicular body protein; Tsg 101, tumour susceptibility gene 101; EEA1, early endosome antigen 1; M6P, mannose 6-phosphate; Atg, autophagy related protein; LC3, microtubule-associated protein light chain 3; UIM, ubiquitin interaction motif; UEV, ubiquitin E2 variant; DUB, deubiquitinating enzyme; PI3K, phosphatidylinositol-3-kinase; UVRAG, UV radiation resistance associated gene; Rab, Ras-related protein in brain; Bcl-2, B-cell lymphoma protein 2; SNARE, N-ethylmaleimide sensitive protein attachment protein receptor; SKD1, suppressor of $\mathrm{K}+$ uptake growth defect; WM, wortmanin

Received 10.10.08; accepted 10.10.08; Edited by G Melino; published online 14.11.08
} 


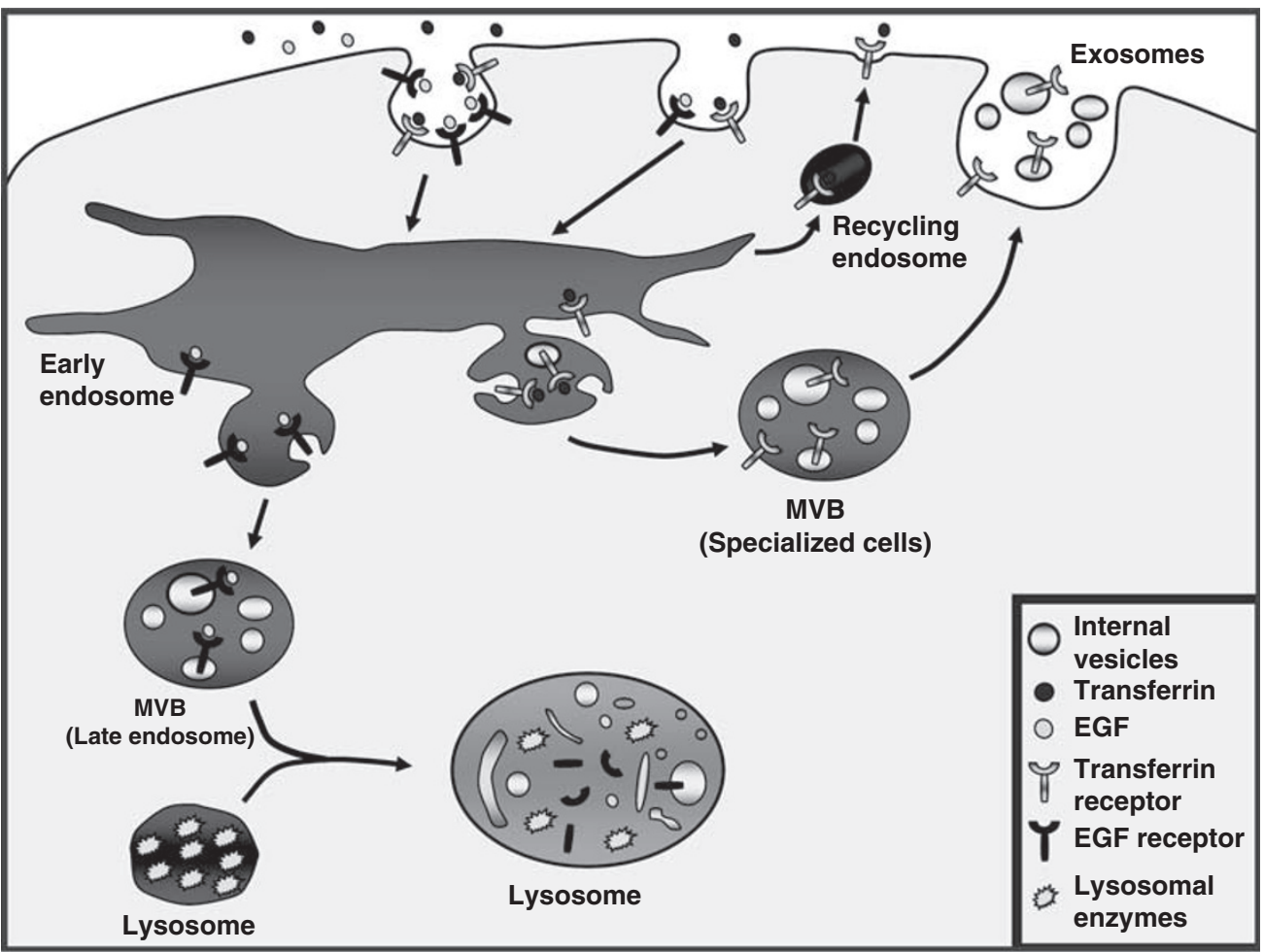

Figure 1 The multivesicular body (MVB) pathway. Endocytosed surface proteins and ligands are delivered into the endosomal system. Several receptors, such as TfR, recycle back to the plasma membrane through recycling endosomes to bind and internalize new ligands. Some cargo proteins destined for degradation in the lysosome, such as EGFR, are sorted into internal lumenal vesicles of MVBs. Mature MVBs can fuse with the lysosome and deliver the internal vesicles to the lysosomal lumen where the vesicles and cargo are degraded by the lysosomal enzymes. In specialized cell types, such as erythroid precursors, some membrane proteins such as the TfR are internalized by endocytosis and selectively sorted into an MVB. Finally, this MVB can fuse with the plasma membrane, releasing the internal vesicles (exosomes) and cargo into the extracellular medium

Several reports have analyzed mainly morphologically the fusion between early and late MVB endosomes with autophagosomes to generate the amphisome. These fusion events indicate that maturation of autophagosome to autophagolysosome, in mammalian cells, is a multistep process. ${ }^{17}$ This convergence between the endocytic and autophagic pathways has been reported in Leydig cells. In this study, the authors have visualized by electron microscopy that MVBs deliver their endocytosed markers (two type of lectins, concanavalin A (ConA) and wheat germ agglutinin (WGA)) to the early autophagosomes where the two pathways meet. ${ }^{18}$

Although the molecular machinery that participates in vesicular trafficking and convergence between both autophagy and MVBs of the endocytic pathway have not been fully uncovered, some proteins involved in this event have been lately elucidated. ${ }^{19}$ This review highlights the most recent advances in our understanding of the molecular machinery that participates in MVB biogenesis and regulates the interaction between autophagy and the MVB route. It is suggested that both autophagy and the MVB pathway have a close relationship with several intracellular events and are implicated in cellular homeostasis.

\section{Biogenesis of MVBs}

Membrane trafficking events are tightly regulated to ensure the correct delivery of cargo. In general, one of the roles of the
MVBs is to recruit mainly transmembrane proteins into its internal vesicles for degradation.

The ubiquitylation process is required for a variety of important biological pathways, including proteosomal degradation, ${ }^{20}$ endocytosis ${ }^{21}$ and lysosomal degradation, ${ }^{22}$ thus regulating the localization and destiny of many proteins. This conjugation event occurs through a sequential series of reactions involving the $\mathrm{E} 1, \mathrm{E} 2$ and $\mathrm{E} 3$ enzymes and results in proteasomal, nuclear and endosomal targeting, depending on the protein and the nature of the ubiquitination. ${ }^{23}$ Although targeting to the proteasome requires attachment of a chain of at least four ubiquitins, monoubiquitination is sufficient for targeting to the endocytic pathway. ${ }^{24,25}$

Several studies have shown that monoubiquitination is the best characterized signal for targeting membrane proteins to the degradative MVB pathway. ${ }^{22,26}$ Most of the transmembrane proteins that accumulate in the internal vesicles of MVBs are marked by a single ubiquitin or a short chain of two or three ubiquitin subunits. These subunits are recognized by a specific and conserved machinery termed 'endosomal sorting complex required for transport' (ESCRT) (for a detailed description of the ESCRT machinery, see Box 1). The ESCRT machinery comprises four separate protein complexes, called ESCRT-0, I, II and III, which have a specific role in sorting proteins into the MVB and in the formation of this kind of endosome (reviewed by Babst, ${ }^{27}$ Nickerson et al., ${ }^{28}$ Saksena et al., ${ }^{29}$ Williams and Urbe $^{30}$ ). 
Box 1 The ESCRT machinery involved in MVB biogenesis

A group of Vps proteins, the endosomal sorting complexes
required for transport (ESCRT)-0, I, II and III, are necessary
for MVB biogenesis. Hrs (hepatocyte growth factor-regulated
tyrosine kinase substrate) or its yeast homolog Vps27 and STAM
(signal-transducing adaptor molecule) or Hse1 in yeast make up
the ESCRT-0 subunit. This complex has an important role in the
initial selection of ubiquitylated cargo at the endosomal membrane
(reviewed by Nickerson et al., ${ }^{28}$ Saksena et al. ${ }^{29}$ and Williams and
Urbe 30 ). ESCRT-I, a 350-kDa complex, is formed by four different
Vps proteins (Vps23, Vps28, Vps37 and Mvb12). Vps23, or its
mammalian homolog Tsg101, is one of the essential subunits of
the ESCRT-I, which recognizes and binds ubiquitylated proteins
through its terminal ubiquitin E2 variant (UEV) domain. Similar to
Tsg101, Hrs is able to bind ubiquitylated proteins because it has
one double-sided ubiquitin interaction motifs (UIMs), which are
important for the sorting of MVB cargo.
domain The Tsg101 UEV
premain interacts with Hrs, through PTVP and PSDP motifs
present in Vps27, allowing the association of ESCRT-I to the
endosomal membrane. The ESCRT-I subunits recruit the
ESCRT-II (Vps22, Vps25 and Vps36), which also has an ubiquitin-
interacting region that is essential for cargo recognition and
sorting. ESCRT-II is able to recruit at least four small proteins to
form an endosomal-associated complex called ESCRT-III (Vps2,
Vps20, Vps24 and Snf7). One of the functions of this complex is
to concentrate the MVB cargo in the inward membrane region.
ESCRT-III also recruits deubiquitinating enzymes (DUBs) to
remove ubiquitin from cargo proteins prior to their incorporation
into the MVB. Finally, ESCRT-III recruits a multimeric AAA
ATPase, Vps4 or its mammalian homolog SKD1, which catalyzes
the disassembly of all ESCRT complexes from the endosomal
membrane in an ATP-dependent manner (reviewed by Babst ${ }^{27}$ ).
This ESCRT disassembly process is the final distinguishable step
of cargo sorting and is an essential event for MVB formation.

Hrs (hepatocyte growth factor-regulated tyrosine kinase substrate), or its yeast homolog Vps27, is a constituent of ESCRT-0, which is necessary for MVB biogenesis (see Box 1). ESCRT-0 has an important role in the initial selection of ubiquitylated cargo at the endosomal membrane. Some reports have shown that Hrs is localized to both early and late endosomal compartments through its FYVE domain, which can bind to the specific lipid phosphatidylinositol-3-phosphate (Ptdlns(3)P). ${ }^{32}$ Interestingly, colocalization studies have shown that localization of the ESCRT-I subunit to the MVB membrane is dependent on its interaction with $\mathrm{Hrs}^{33,34}$ Moreover, Hrs might bind clathrin through its clathrin-binding motif. Interestingly, the colocalization of Hrs with endosomal clathrin coats has been demonstrated by electron microscopy. ${ }^{26,30}$

A recent publication has demonstrated that inhibition of Hrs or the different subunits of ESCRT-I and II, but not Vps24 (a component of ESCRT-III subunit) caused ubiquitylated proteins to accumulate in enlarged MVBs. ${ }^{35}$ On the other hand, all of the ESCRT subunits remain tightly associated with the membrane in the absence of Vps4 (a multimeric AAA ATPase), thus blocking cargo sorting and generating an enlarged type of vesicles termed class E phenotype. ${ }^{28,36}$ This result suggests that Vps4 activity is necessary for cargo transport and for removal of the ESCRT machinery from the endosomal membrane. However, even though dissociation of ESCRT-III is essential for sorting, it is not a prerequisite for luminal vesicle formation, ${ }^{37}$ and for uncoupling MVB cargo sorting from the budding of vesicles into the endosomal lumen.
Alix, another class E Vps protein, has been shown to regulate $M V B$ biogenesis (for a review, see Lakkaraju and Rodriguez-Boulan ${ }^{38}$ ). Alix is able to interact with CHMP4, which is homologous to ESCRT-III subunits Vps32/Snf7 and Tsg101. $^{39}$ It has been demonstrated that Bro1, a Saccharomyces cerevisiae Alix homolog, recruits the ubiquitin thiolesterase Doa4 (a DUB implicated in MVB pathway) to the endosomal membrane where it might regulate cycles of ubiquitylation/deubiquitination. ${ }^{39-42}$ As mentioned above, this cycle is essential for sorting proteins into MVBs.

In summary, the ubiquitylation system has an important role in protein sorting into MVBs. However, recent works have demonstrated that the cargo itself can drive MVB biogenesis and proteins can be sorted into MVBs in a ubiquitin- ${ }^{43,44}$ and ESCRT-independent manner. ${ }^{45}$

\section{Autophagy}

Autophagy, is a lysosomal degradation pathway for cytoplasmic components (for a review, see Klionsky and Emr, ${ }^{10}$ Eskelinen ${ }^{17}$ and Theos et al. ${ }^{46}$ ). Unlike the ubiquitin-proteasome degradation system responsible for degradation of short-lived proteins, autophagy is involved in the turnover of long-lived proteins, cellular macromolecules and whole organelles, ${ }^{13,47-50}$ acting as an essential physiological system for cellular homeostasis. ${ }^{51}$ It has also been demonstrated that autophagy has an important role in neurodegenerative diseases, aging, cancer, type-Il programmed cell death, antigen presentation, early neonatal stages and bacterial invasion (for a review, see Tanida et al. ${ }^{11}$ ).

During the autophagic process, a cupped-shape structure, also referred to as phagophore, isolation membrane or pre-autophagosome, encloses a portion of cytoplasm, forming an autophagosome. The autophagosome is a doublemembraned structure containing undigested cytoplasmic components, including organelles, and it is devoid of any lysosomal protein. These autophagosomes can interact with components of the endocytic pathway, generating a hybrid organelle termed amphisome. ${ }^{17,52-55}$ Studies in rat liver indicate that amphisomes are enriched in proteins of early endosomes, such as EEA1, and also of late endosomes (M6P receptor) and, it is therefore likely that amphisomes receive cargo proteins from both early (sorting) and late (MVB) endosomes. ${ }^{12}$ The amphisome is more acidic than the autophagosome ( $\mathrm{pH} 5.7)$, probably due to the fusion of autophagosomes with endocytic vesicles carrying the proton pump and lysosomal membrane proteins, but not lysosomal enzymes. ${ }^{12,17}$ Finally, the autophagosome or the amphisome acquires hydrolytic enzymes by fusion with lysosomes (to form the autolysosome), in which intraluminal components and the inner membrane of the autophagosome is degraded $^{13,56}$ (see Figure 2).

The molecular mechanism underlying autophagy was first characterized in yeast. These studies led to the identification of a set of Atg (autophagy related) factors, which are evolutionary conserved. ${ }^{10,56,57}$ Two ubiquitin-like conjugation systems that participate in a coordinated manner are necessary in early stages of autophagosome formation ${ }^{58-60}$ (see Box 2). The first conjugation system produces the Atg12Atg5 complex that is non-covalently associated with Atg16. 


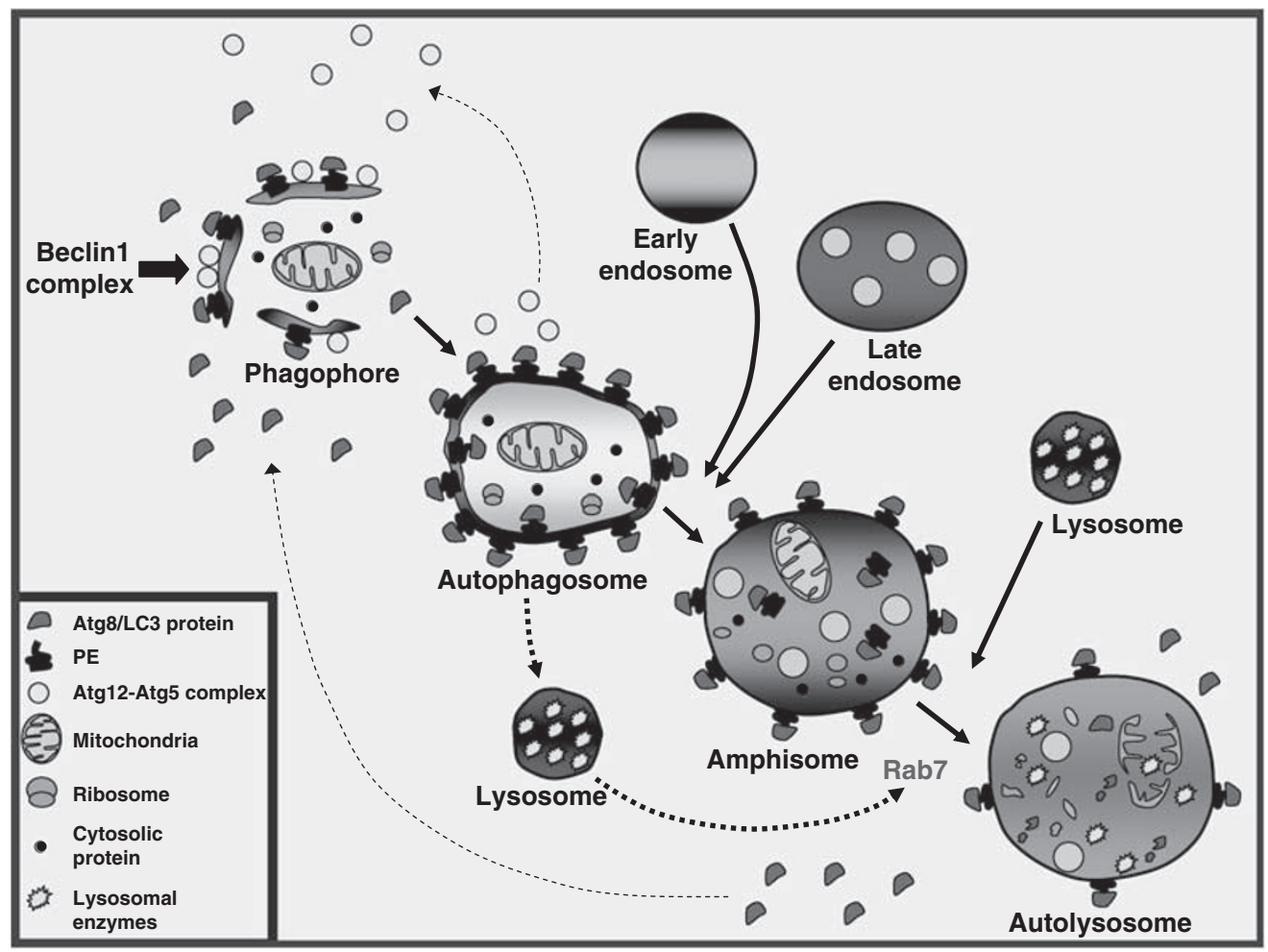

Figure 2 The autophagic pathway. The production of PtIns3P by the Beclin 1 complex is an early event in autophagosome formation that is necessary for the recruitment of certain Atg proteins to the phagophore or isolation membrane. Subsequently, the sequential recruitment of Atg12-Atg5 complex and Atg8-LC3 protein to the phagophore takes place. The cytosolic form of LC3, LC3-I, is covalently bound to phosphatidylethanolamine (PE), generating LC3-II, which binds to the autophagosomal membrane. The phagophore, a cup-shaped structure, encapsulates cytosolic proteins and organelles, forming a double-membraned structure termed autophagosome. At this point, most of the Atg proteins, except LC3-II, are recycled from the autophagosome membrane back to the cytosol. Then, the autophagosome can fuse with endosomal compartments, generating the amphisome. The sequestered material is finally degraded by fusion of the amphisome with the lysosomal compartment, forming the autolysosome. Fusion of autophagosomes and amphisomes with the lysosomal compartment depends on an active Rab7

The second conjugation system is characterized by conjugation of Atg8 or its mammalian homolog LC3 (see Box 2) with phosphatidylethanolamine (Figure 3).

\section{Regulation of Autophagy}

In mammalian cells, different extracellular signals can trigger autophagy such as nutrient starvation, stress or treatment with hormones. ${ }^{62,63}$ One target of these signals is the serine threonine protein kinase Tor (Target of rapamycin), a phosphorylated kinase that inhibits the autophagic process until it is dephosphorylated. The first evidence that Tor has a role in regulating autophagy came from experiments involving rat hepatocytes that showed that rapamycin partially reverses the inhibitory effects of amino acids on autophagic proteolysis. ${ }^{64}$ The protein Tor functions as a sensor for cellular energy and amino-acid levels. ${ }^{65}$

As mentioned above, one of the most important physiological stimuli that favor the activation of autophagy is nutrient starvation such as deprivation of serum and some amino acids (leucine, phenylalanine, tyrosine, glutamine and alanine). ${ }^{66,67}$ Earlier study has also shown that a class III phosphatidylinositol-3-kinase (PI3K)/Vps34 is involved in the early steps prior to autophagosome formation. ${ }^{68,69}$ As expected, inhibitors of PI3Ks such as wortmannin (WM), 3-methyladenine and LY294002 inhibit autophagy. ${ }^{70}$ Thus, $^{2}$ the levels of autophagy can also be modulated pharmacologically. ${ }^{71}$ Several reports have shown the essential role of $\mathrm{PI3K} /$ ps34 at the pre-autophagosomal structure in the recruitment of proteins necessary for the biogenesis of the membrane-bound compartment.

Beclin 1, the mammalian ortholog of yeast Atg6/Vps30, is an important link between the autophagy pathway and apoptosis. ${ }^{49}$ Beclin 1 binds to the mammalian homolog of PI3K/Vps34 and this interaction is evolutionarily conserved. This complex is essential for the recruitment of other Atg proteins to the pre-autophagosomal structure (phagophore), ${ }^{72,73}$ and for the production of Ptdlns3P. In yeast, Atg6 and Vps34 can form part of two different protein complexes. One of them is implicated in vacuolar protein sorting, whereas the other one modulates autophagy under starvation conditions. ${ }^{74}$ UVRAG is a tumor suppressor that is part of the Beclin 1 complex, and stimulates autophagy through a direct interaction with the coiled-coil domain of Beclin 1. It is important to mention that UVRAG colocalizes not only with autophagosomes but also with early endosomes. ${ }^{75}$ UVRAG has a key role in endocytic trafficking, promoting endosomal fusion both in vitro and in vivo. It is required for EGF trafficking, favoring the degradation of EGFR. Interestingly, it has been found that UVRAG also 
Box 2 The protein conjugation systems involved in the autophagy pathway

As shown in Figure 3, autophagosome formation is mediated by a group of evolutionarily conserved Atg proteins (autophagy-related proteins). For many of these genes, the corresponding orthologs have been identified in higher eukaryotes, including man. Two protein conjugation systems, which are similar to those involved in the ubiquitylation of proteins in the proteasome pathway and function in a coordinated manner, are involved in the autophagy pathway. The first conjugation system requires the proteins Atg5, Atg7, Atg10 and Atg12; Atg7 and Atg10 play the role of E1 and E2 enzymes of the ubiquitin pathway, respectively. Finally, the complex Atg5-Atg12 is formed in association with Atg16 in a noncovalent form. Interestingly, a recent publication has shown that the Atg12-Atg5 conjugate has E3-like activity for the Atg8-PE (phosphatidylethanolamine) conjugation reaction. ${ }^{61}$ This multimeric complex is associated with the membrane, where it initiates the formation of the phagophore or isolation membrane. The other conjugation system involves the Atg8 protein or its mammalian homolog MAP-LC3 (microtubule-associated protein 1 light chain 3). On synthesis, LC3 is processed by a cysteine protease to its cytosolic form LC3-I, exposing a glycine residue at the carboxy terminus. This form is conjugated to the lipid PE, generating the LC3-II form, which is associated with both the outer and inner autophagosomal membranes (Figure 2). This conjugation event is dependent on the activity of Atg7 and Atg3, which have a similar function to the E1 and E2 enzymes of the ubiquitin pathway, respectively. Then, autophagosomes and amphisomes can fuse with lysosomes to degrade the trapped components, generating the autophagolysosome. Finally, the cysteine protease Atg4 is involved in the processing and deconjugation of LC3-II from the autophagosome, although LC3-II is also present in late autophagic vesicles (i.e.

autophagolysosomes) at lesser concentrations. In general, LC3 processing and distribution is a useful tool to monitor autophagy either morphologically or biochemically.

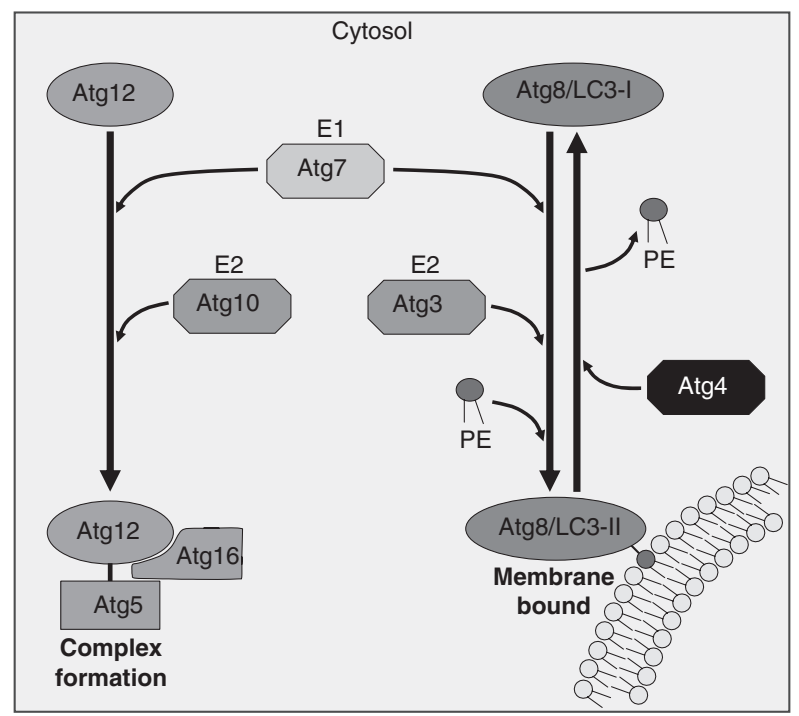

Figure 3 Schematic representation of the ubiquitin-like systems necessary for autophagy. Two ubiquitin-like systems are required for autophagy. Atg12 is activated by Atg7 (an E1 enzyme), transferred to Atg10 (an E2 enzyme) and conjugated to Atg5, later forming a complex with Atg16. This complex is the first modifier necessary for the development of precursors of autophagosomes. The second modifier necessary for autophagosome formation is Atg8/LC3, which is synthesized as a pro-LC3 and processed to LC3-I (cytosolic form). LC3-I is activated by Atg7, transferred to Atg3 (an E2 enzyme) and finally conjugated with phosphatidylethanolamine (PE), generating the membrane bound form LC3-II. LC3II is converted to LC3-I by the cysteine protease Atg 4 interacts with the early endosomal protein Rab5, indicating again the connection between autophagy and the endocytic pathway.

Some proteins involved in the endolysosomal pathway are required for autophagosome maturation in yeast and mammalian cells, such as the small GTPase Rab7/Ypt7p, ${ }^{76,77}$ the small GTPase Rab11, ${ }^{19}$ a syntaxin homolog Vam3p, the AAA ATPase SKD1 and the SNARE protein Vti1p. Furthermore, in a very recent publication it has been demonstrated that the early endosome protein Rab5, in a complex with Beclin 1 and $\mathrm{PI} 3 \mathrm{~K} / \mathrm{Vps} 34$, is required at an initial stage of the autophagosome formation. ${ }^{78}$ Thus, the interplay between the endocytic and autophagic pathways at the molecular level is much closer than envisioned earlier.

\section{Relationship between Autophagy and the MVB Pathway: Molecular Players}

As indicated above, biochemical and morphological studies have shown that autophagosomes can fuse with endosomes, forming the so-called amphisome. This structure represents a prelysosomal compartment in which both the autophagic and endocytic pathways converge. ${ }^{12,17}$ It has been demonstrated that amphisomes can be distinguished physically and morphologically from other closely related autophagic and endocytic vacuoles. ${ }^{12}$ Leupeptin (an inhibitor of intralysosomal proteolysis) was used as a tool to accumulate amphisomes in rat liver cells. Morphometric analysis indicated that the amphisome is an independent prelysosomal endocytic structure that can be distinguished from other endocytic vacuoles. $^{12}$

Several lines of evidence indicate that autophagosomeendosome interaction is required for the maturation of the autophagosome. Interestingly, morphological evidence suggests that MVBs are the main endocytic fusion partners that meet with the autophagosome. ${ }^{12}$ However, there is also good evidence that some autophagosomes can fuse directly with lysosomes, without amphisome formation. Morphometric studies have shown that hepatocytic endosomes were 5-6 times more likely to enter the amphisome pathway than to fuse directly with lysosomes. ${ }^{52}$

In general, very little is known about the molecular components that actually regulate the interaction between the autophagosome and the endocytic/MVB pathway. Recent evidence indicates that calcium is one of the players. Calcium is an important element that plays an essential role in many biological processes (reviewed by Ding and Yin $^{79}$ and HoyerHansen and Jaattela ${ }^{80}$ ). It was initially shown that autophagy was dependent on calcium. ${ }^{81}$ Recent work has demonstrated the important role of calcium in autophagosome formation, pointing to $\mathrm{Bcl}-2$ as a possible modulator of calcium homeostasis and signaling. ${ }^{82,83}$ Calcium-mobilizing agents, such as ionomycin and thapsigargin, cause an increase in free cytosolic calcium, inducing autophagy through a signaling pathway that involves calmodulin-dependent kinase kinase $\beta$ and AMP-activated protein kinase. ${ }^{82}$ The effect of these calcium-mobilizing agents was blocked by the calcium chelator BAPTA-AM. Moreover, a membrane-permeable fluorescent calcium probe has recently been used to demonstrate that, under conditions that stimulate autophagy, 
autophagosomes accumulate calcium intralumenally; thus, the autophagosome and amphisome actually act as a calcium store. The importance of this finding is that, similar to other intracellular fusion events and exocytic processes, the intravesicular accumulation of calcium and likely local calcium efflux seems to be required for autophagosome fusion. The calcium chelator BAPTA-AM abrogated autophagosome enlargement and autophagosome-MVB fusion, ${ }^{19}$ suggesting that both autophagosome homotypic fusion and fusion with other compartments are calcium dependent. Collectively, these results imply that the accumulation of calcium in autophagic vacuoles facilitates the interaction with MVBs. It has been demonstrated that fusion among early endosomes also requires release of intravesicular calcium. In current models it is postulated that, during a vesicular fusion event, the membranes of each compartment are maintained in close proximity, generating a partially restricted environment in which the calcium concentration can be strongly increased by the local action of calcium pumps or the opening of calcium channels in the vesicles engaged in fusion. The emptiness of these vesicular stores, due to the release of calcium, could subsequently open store-operated calcium channels in the plasma membrane, enabling calcium to enter from the extracellular medium. As part of the fusion machinery (together with the SNAREs), the calcium sensor synaptotagmin is recruited to the membrane on calcium binding, leading to the final fusion step.

As indicated in the previous sections, in specialized cell types, such as reticulocytes, MVBs can fuse with the plasma membrane, releasing the internal vesicles, the so-called exosomes, into the extracellular media. Human erythroleukemic K562 cells generate MVBs and have been used to study the participation of molecular regulators in the MVB pathway and exosome release.$^{84,85} \mathrm{~K} 562$ cells are an useful tool to study MVB formation and exosome secretion from erythroid cells because unlike reticulocytes, it is possible to generate transient or stably transfected cell lines overexpressing different proteins involved in membrane trafficking. Furthermore, given the importance of both the MVB and autophagic pathways in reticulocyte maturation, the $\mathrm{K} 562$ cells are an excellent model to study the interaction of these pathways as well as the consequence of activating autophagy in this interplay.

We have demonstrated that in K562 cells overexpression of wild-type Rab11 and its active mutant Rab11Q70L generates large MVBs decorated by Rab11, stimulating exosome release. ${ }^{84,85}$ It is noteworthy that the stimulatory effect of Rab11 in exosome secretion was over-ridden by overexpression of the autophagic protein LC3. In addition, induction of autophagy by nutrient deprivation inhibited exosome secretion, suggesting that autophagy induction re-routes the MVBs toward the autophagic pathway, hampering exosome release. ${ }^{19}$ We have presented evidence that physiological or pharmacological induction of autophagy caused a marked increase in the fusion between MVBs and autophagosomes, generating enlarged amphisomes. Interestingly, overexpression of the Rab11 dominant negative mutant impaired autophagosome-MVB interaction, suggesting that Rab11 is necessary for this fusion event. ${ }^{19}$ Likewise, fusion between autophagosomes and MVBs was partially retarded in hepa- tocytes from mice deficient in the SNARE protein Vti1p, suggesting that this protein also participate in autophagosome-MVB fusion in mammalian cells. ${ }^{86}$ On the other hand, Vti1 might have a role in autophagic vacuole maturation. ${ }^{17}$ Recent publications demonstrated that the small GTPase Rab7, which is localized in the limiting membrane of autophagosome, is important for the normal progression of autophagy. ${ }^{51,76,77}$ Interestingly, we have also shown that Rab7 is necessary for fusion between autophagosome and amphisomes with the lysosome, whereas it is not required for autophagosome-MVB fusion. ${ }^{19}$ Moreover, we have shown that inhibition of the interaction between autophagosomes and MVBs, to generate the amphisome, does not hamper the autophagolysosome formation, supporting the existence of an alternative direct fusion event between autophagosomes and lysosomes.

Earlier studies have shown that vinblastine, a microtubuledisrupting agent, prevents the interaction between autophagosomes and lysosomes. It has been demonstrated that this is due to inhibition of fusion of autophagosomes and amphisomes with lysosomes. ${ }^{12,15}$ In contrast, in a recent publication it has been shown that microtubule depolymerization does not affect the rate of autolysosome formation. ${ }^{87}$ Thus, the exact blocking point between the endocytic and autophagy pathways is by some means debatable. We have shown that the interaction between MVBs and autophagic vesicles was not prevented when microtubules were depolymerized by vinblastine but fusion with lysosomes was completely hampered. ${ }^{19}$ Consistent with our observations, Tooze and collaborators ${ }^{88}$ have shown that microtubuledepolymerizing agents inhibit fusion of autophagosomes with lysosomal vacuoles. In summary, all these studies indicate that microtubules are not required at the autophagosomeMVB fusion step, but are necessary for the final maturation and degradation stages.

The AAA ATPase SKD1 was also proposed as an essential protein for autophagosome maturation. ${ }^{89}$ Interestingly, overexpression of a negative mutant of SKD1 (SKD1 E235Q) that is unable to hydrolyze ATP induced aberrant MVBs. ${ }^{89}$ Furthermore, overexpression of this mutant blocks the autophagic pathway at a step prior to fusion with the lysosome, resulting in a decrease in protein degradation. These data suggest that SKD1 is necessary for both endosomal vesicle trafficking and a late stage of the autophagic pathway that is necessary for autophagosome maturation.

As indicated above, PI3Ks are key enzymes that regulate the autophagic pathway. Type III PI3K/Vps34 is thought to be involved in the early steps of this pathway as the PI3K inhibitor WM completely blocks autophagy at a step prior to the formation of the autophagosomal-sequestering vesicle. Moreover, it is known that a PI3K also participates in the biogenesis of MVBs; its activity is required for the generation of the internal vesicles. An interesting observation is that in $\mathrm{K} 562$ cells the LC3 protein has a punctate distribution even in cells incubated in full nutrient conditions, indicating that autophagic vesicles are already formed. ${ }^{90}$ Nevertheless, these LC3labeled structures disappeared on incubation with the autophagy inhibitor WM, indicating that their distribution was indeed regulated by a PI3K; this is consistent with the requirement for this enzyme in autophagosome formation 


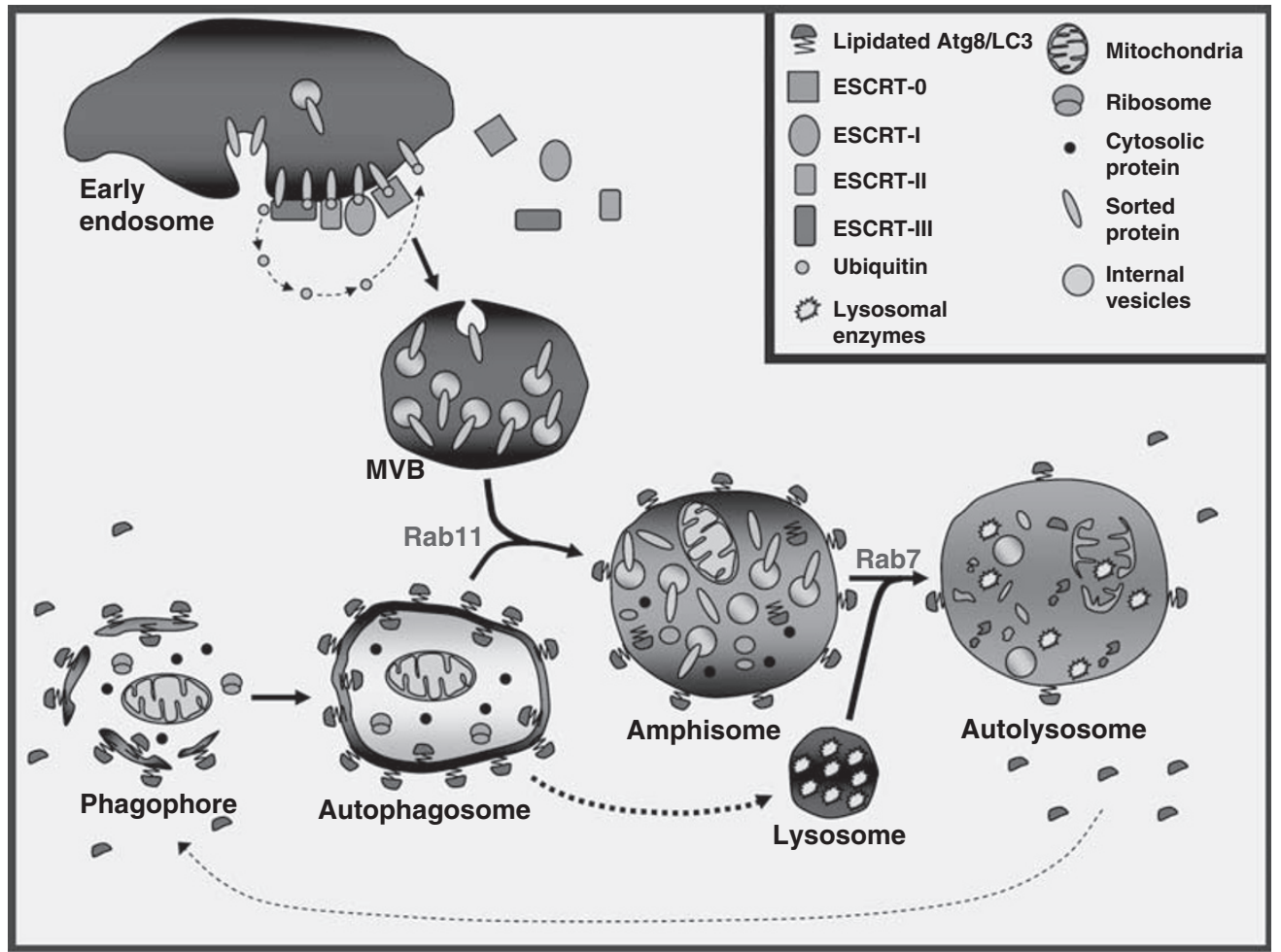

Figure 4 Convergence of autophagic vesicles and MVBs destined for lysosomal protein degradation. The model shows that organelles and cytosolic proteins that are targeted for degradation are engulfed in a double membrane vacuole (phagophore or isolation membrane), generating an autophagosome. In the endocytic pathway, cargo proteins destined for lysosomal degradation are sorted into lumenal vesicles of MVBs. ESCRT-0, I, II and III are recruited from the cytoplasm to the endosomal membrane likely in a sequential manner, enabling the inward budding of the early endosome-limiting membrane. Mature MVBs fuse with the autophagosome (a Rab11-dependent step) forming a hybrid organelle - the so-called amphisome. Finally, amphisomes can fuse with the lysosome (the enzyme-containing compartment) generating the autophagolysosome, a Rab7-dependent event

(for a review, see Meijer and Codogno ${ }^{50}$ ). As expected, WM treatment completely prevented the formation of MVBs, although giant vesicles were generated due to the inhibition of inward budding as demonstrated earlier (Fader et al., unpublished).

Beclin 1 forms a complex with $\mathrm{PI} 3 \mathrm{~K} / \mathrm{Vps} 34$. Earlier report ${ }^{91}$ used siRNA-mediated gene silencing methodology to suppress Beclin 1 expression. It was demonstrated that whereas autophagy was markedly attenuated, no effect was observed in other PI3K-dependent pathways, such as endocytic trafficking or lysosomal enzyme sorting, suggesting that Beclin 1 is a restricted modulator of autophagy. However, we have observed that knocking down Beclin 1 expression to specifically affect the PI3K involved in the autophagy pathway ${ }^{91}$ not only hampered LC3 processing under both control and starvation conditions but also altered the distribution and the size of the MVBs (Fader et al., unpublished). This result suggests that Beclin 1, an autophagic protein, also participates in MVB formation.

Several diseases are associated with autophagy. Neurodegenerative disorders (Huntington's and Parkinson's) are characterized by accumulation of intracellular protein aggregates. Autophagy is activated as an alternative lysosomal mechanism to degrade these protein aggregates. ${ }^{92-95}$ In addition, CHMP2B/Vps2B, a subunit of the ESCRT-III complex, was found to be mutated in patients with fronto- temporal dementia) and amyotrophic lateral sclerosis. ${ }^{96,97}$ Recent publications have shown that autophagy degradation is abrogated in cells depleted of ESCRT subunits or in cells overexpressing a mutant of CHMP2B that caused an accumulation of ubiquitin-protein aggregates. ${ }^{35,98}$ Indeed, loss of mSnf7-2, a key component of ESCRT-III that is highly expressed in neurons, caused autophagosome accumulation in cortical neurons, retraction of dendrites and neuronal cell loss. ${ }^{98}$ Likewise, loss of ESCRT or Vps4 activity in Drosophila caused accumulation of autophagosomes, probably due to inhibition of fusion with compartments of the endocytic pathway. ${ }^{99}$ Furthermore, this ESCRT dysfunction increased the toxicity of polyglutamine aggregates in a model for Huntington's disease. ${ }^{99}$ Interestingly, the ESCRT machinery has been shown to have an important and conserved function in modulating bacterial phagosome in mammalian cells. ${ }^{100}$ In summary, all these recent reports link ESCRT activity with autophagy and neurodegenerative diseases or other pathological processes.

Although little is still known about the proteins that participate in the fusion of autophagosomes and endosomes, in particular MVBs, cumulative evidence suggests that a close relationship between the MVB and autophagic pathways exist (see Figure 4). In fact, alteration in MVB biogenesis blocks the degradation of some proteins through autophagy (see also a recent review by Rusten and Simonsen ${ }^{101}$ ). In summary, 
these results indicate that a functional MVB is necessary to allow an efficient autophagic degradation. Further experiments would allow us to decipher the connection between both pathways at the molecular level. Given the critical role of MVB biogenesis and autophagy in many diseases, it is clear that future research in this field will greatly improve our knowledge and suggest therapeutic intervention possibilities.

Acknowledgements. Our study in this area is funded by grants from Agencia Nacional de Promoción Científica y Tecnológica (PICT2004 no. 20711 and PICT 2005 no. 38420), CONICET (PIP no. 5943) and SECTyP (Universidad Nacional de Cuyo).

1. Mellman I. Membranes and sorting. Curr Opin Cell Biol 1996; 8: 497-498.

2. Harding $C$, Heuser J, Stahl P. Receptor-mediated endocytosis of transferrin and recycling of the transferrin receptor in rat reticulocytes. J Cell Biol 1983; 97: 329-339.

3. Harding C, Heuser J, Stahl P. Endocytosis and intracellular processing of transferrin and colloidal gold-transferrin in rat reticulocytes: demonstration of a pathway for receptor shedding. Eur J Cell Biol 1984; 35: 256-263.

4. Johnstone RM, Mathew A, Mason AB, Teng K. Exosome formation during maturation of mammalian and avian reticulocytes: evidence that exosome release is a major route for externalization of obsolete membrane proteins. J Cell Physiol 1991; 147: 27-36.

5. Johnstone RM. The Jeanne Manery-Fisher Memorial Lecture 1991. Maturation of reticulocytes: formation of exosomes as a mechanism for shedding membrane proteins. Biochem Cell Biol 1992; 70: 179-190.

6. Johnstone RM. Cleavage of the transferrin receptor by human granulocytes: differentia proteolysis of the exosome-bound TFR. J Cell Physiol 1996; 168: 333-345.

7. Thery C, Zitvogel L, Amigorena S. Exosomes: composition, biogenesis and function. Nat Rev Immunol 2002; 2: 569-579.

8. Gronowicz G, Swift H, Steck TL. Maturation of the reticulocyte in vitro. J Cell Sci 1984; 71 177-197.

9. Dunn Jr WA. Studies on the mechanisms of autophagy: maturation of the autophagic vacuole. J Cell Biol 1990; 110: 1935-1945.

10. Klionsky DJ, Emr SD. Autophagy as a regulated pathway of cellular degradation. Science 2000; 290: 1717-1721.

11. Tanida I, Minematsu-lkeguchi N, Ueno T, Kominami E. Lysosomal turnover, but not a cellular level, of endogenous LC3 is a marker for autophagy. Autophagy 2005; 1: 84-91.

12. Berg TO, Fengsrud M, Stromhaug PE, Berg T, Seglen PO. Isolation and characterization of rat liver amphisomes. Evidence for fusion of autophagosomes with both early and late endosomes. J Biol Chem 1998; 273: 21883-21892.

13. Dunn Jr WA. Autophagy and related mechanisms of lysosome-mediated protein degradation. Trends Cell Biol 1994; 4: 139-143.

14. Gordon PB, Seglen PO. Prelysosomal convergence of autophagic and endocytic pathways. Biochem Biophys Res Commun 1988; 151: 40-47.

15. Gordon PB, Hoyvik H, Seglen PO. Prelysosomal and lysosomal connections between autophagy and endocytosis. Biochem J 1992; 283 (Part 2): 361-369.

16. Shintani T, Klionsky DJ. Autophagy in health and disease: a double-edged sword. Science 2004; 306: 990-995.

17. Eskelinen EL. Maturation of autophagic vacuoles in mammalian cells. Autophagy 2005; 1: $1-10$.

18. Yi J, Tang XM. The convergent point of the endocytic and autophagic pathways in Leydig cells. Cell Res 1999; 9: 243-253.

19. Fader CM, Sanchez D, Furlan M, Colombo Ml. Induction of autophagy promotes fusion of multivesicular bodies with autophagic vacuoles in k562 cells. Traffic 2008; 9 . 230-250.

20. Hershko A. Mechanisms and regulation of the degradation of cyclin B. Philos Trans $R$ Soc Lond B Biol Sci 1999; 354: 1571-1575.

21. Hicke L, Dunn R. Regulation of membrane protein transport by ubiquitin and ubiquitinbinding proteins. Annu Rev Cell Dev Biol 2003; 19: 141-172.

22. Katzmann DJ, Odorizzi G, Emr SD. Receptor downregulation and multivesicular-body sorting. Nat Rev Mol Cell Biol 2002; 3: 893-905.

23. Teo H, Veprintsev DB, Williams RL. Structural insights into endosomal sorting complex required for transport (ESCRT-I) recognition of ubiquitinated proteins. J Biol Chem 2004 279: 28689-28696.

24. Chau V, Tobias JW, Bachmair A, Marriott D, Ecker DJ, Gonda DK et al. A multiubiquitin chain is confined to specific lysine in a targeted short-lived protein. Science 1989; 243 1576-1583.

25. Thrower JS, Hoffman L, Rechsteiner M, Pickart CM. Recognition of the polyubiquitin proteolytic signal. EMBO J 2000; 19: 94-102.

26. Raiborg C, Rusten TE, Stenmark H. Protein sorting into multivesicular endosomes. Curr Opin Cell Biol 2003. 15: 446-455.

27. Babst M. A protein's final ESCRT. Traffic 2005; 6: 2-9
28. Nickerson DP, Russell MR, Odorizzi G. A concentric circle model of multivesicular body cargo sorting. EMBO Rep 2007; 8: 644-650.

29. Saksena S, Sun J, Chu T, Emr SD. ESCRTing proteins in the endocytic pathway. Trends Biochem Sci 2007; 32: 561-573.

30. Williams RL, Urbe S. The emerging shape of the ESCRT machinery. Nat Rev Mol Cell Biol 2007; 8: 355-368

31. Raiborg C, Stenmark H. Hrs and endocytic sorting of ubiquitinated membrane proteins. Cell Struct Funct 2002; 27: 403-408.

32. Gaullier JM, Simonsen A, D'Arrigo A, Bremnes B, Stenmark H, Aasland R. FYVE fingers bind Ptdlns(3)P. Nature 1998; 394: 432-433.

33. Bache KG, Brech A, Mehlum A, Stenmark $H$. Hrs regulates multivesicular body formation via ESCRT recruitment to endosomes. J Cell Biol 2003; 162: 435-442.

34. Katzmann DJ, Stefan CJ, Babst M, Emr SD. Vps27 recruits ESCRT machinery to endosomes during MVB sorting. J Cell Biol 2003; 162: 413-423.

35. Filimonenko M, Stuffers S, Raiborg C, Yamamoto A, Malerod L, Fisher EM et al. Functional multivesicular bodies are required for autophagic clearance of protein aggregates associated with neurodegenerative disease. J Cell Biol 2007; 179: 485-500.

36. Azmi I, Davies B, Dimaano C, Payne J, Eckert D, Babst M et al. Recycling of ESCRTs by the AAA-ATPase Vps4 is regulated by a conserved VSL region in Vta1. J Cell Biol 2006; 172: 705-717.

37. Nickerson DP, West M, Odorizzi G. Did2 coordinates Vps4-mediated dissociation of ESCRT-III from endosomes. J Cell Biol 2006; 175: 715-720.

38. Lakkaraju A, Rodriguez-Boulan E. Cell biology: caught in the traffic. Nature 2007; 448: 266-267.

39. Sadoul R. Do Alix and ALG-2 really control endosomes for better or for worse? Biol Cell 2006; 98: 69-77.

40. Luhtala N, Odorizzi G. Bro1 coordinates deubiquitination in the multivesicular body pathway by recruiting Doa4 to endosomes. J Cell Biol 2004; 166: 717-729.

41. Nikko E, Marini AM, Andre B. Permease recycling and ubiquitination status reveal a particular role for Bro1 in the multivesicular body pathway. J Biol Chem 2003; 278. 50732-50743.

42. Odorizzi G, Katzmann DJ, Babst M, Audhya A, Emr SD. Bro1 is an endosome-associated protein that functions in the MVB pathway in Saccharomyces cerevisiae. J Cell Sci 2003; 116: 1893-1903.

43. Reggiori F, Pelham HR. Sorting of proteins into multivesicular bodies: ubiquitindependent and -independent targeting. EMBO J 2001; 20: 5176-5186.

44. White IJ, Bailey LM, Aghakhani MR, Moss SE, Futter CE. EGF stimulates annexin 1-dependent inward vesiculation in a multivesicular endosome subpopulation. EMBO J 2006; 25: 1-12.

45. Theos AC, Truschel ST, Tenza D, Hurbain I, Harper DC, Berson JF et al. A lumenal domain-dependent pathway for sorting to intralumenal vesicles of multivesicular endosomes involved in organelle morphogenesis. Dev Cell 2006; 10: 343-354.

46. Mizushima N, Ohsumi $\mathrm{Y}$, Yoshimori T. Autophagosome formation in mammalian cells. Cell Struct Funct 2002; 27: 421-429.

47. Bohley P, Seglen PO. Proteases and proteolysis in the lysosome. Experientia 1992; 48: 151-157.

48. Klionsky DJ, Ohsumi Y. Vacuolar import of proteins and organelles from the cytoplasm. Annu Rev Cell Dev Biol 1999; 15: 1-32.

49. Levine B, Klionsky DJ. Development by self-digestion: molecular mechanisms and biological functions of autophagy. Dev Cell 2004; 6: 463-477.

50. Meijer AJ, Codogno P. Regulation and role of autophagy in mammalian cells. Int J Biochem Cell Biol 2004; 36: 2445-2462.

51. Kimura S, Noda T, Yoshimori T. Dissection of the autophagosome maturation process by a novel reporter protein, tandem fluorescent-tagged LC3. Autophagy 2007; 3: 452-460.

52. Liou W, Geuze HJ, Geelen MJ, Slot JW. The autophagic and endocytic pathways converge at the nascent autophagic vacuoles. J Cell Biol 1997; 136: 61-70.

53. Lucocq J, Walker D. Evidence for fusion between multilamellar endosomes and autophagosomes in HeLa cells. Eur J Cell Biol 1997; 72: 307-313.

54. Punnonen EL, Autio S, Kaija $\mathrm{H}$, Reunanen $\mathrm{H}$. Autophagic vacuoles fuse with the prelysosomal compartment in cultured rat fibroblasts. Eur J Cell Biol 1993; 61: 54-66.

55. Tooze J, Hollinshead M, Ludwig T, Howell K, Hoflack B, Kern H. In exocrine pancreas, the basolateral endocytic pathway converges with the autophagic pathway immediately after the early endosome. J Cell Biol 1990; 111: 329-345.

56. Mizushima N, Yamamoto A, Hatano M, Kobayashi Y, Kabeya Y, Suzuki K et al. Dissection of autophagosome formation using Apg5-deficient mouse embryonic stem cells. J Cell Biol 2001; 152: 657-668.

57. Klionsky DJ. The molecular machinery of autophagy: unanswered questions. J Cell Sci 2005; 118: 7-18.

58. Nemoto T, Tanida I, Tanida-Miyake E, Minematsu-lkeguchi N, Yokota M, Ohsumi M et al. The mouse APG10 homologue, an E2-like enzyme for Apg12p conjugation, facilitates MAP-LC3 modification. J Biol Chem 2003; 278: 39517-39526.

59. Suzuki K, Kirisako T, Kamada Y, Mizushima N, Noda T, Ohsumi Y. The preautophagosomal structure organized by concerted functions of APG genes is essential for autophagosome formation. EMBO J 2001; 20: 5971-5981.

60. Tanida I, Nishitani T, Nemoto T, Ueno T, Kominami E. Mammalian Apg12p, but not the Apg12p.Apg5p conjugate, facilitates LC3 processing. Biochem Biophys Res Commun 2002; 296: 1164-1170. 
61. Hanada T, Noda NN, Satomi Y, Ichimura Y, Fujioka Y, Takao T et al. The Atg12-Atg5 conjugate has a novel E3-like activity for protein lipidation in autophagy. J Biol Chem 2007; 282: 37298-37302.

62. Mortimore GE, Schworer CM. Induction of autophagy by amino-acid deprivation in perfused rat liver. Nature 1977; 270: 174-176.

63. Mortimore GE, Poso AR. Intracellular protein catabolism and its control during nutrient deprivation and supply. Annu Rev Nutr 1987; 7: 539-564.

64. Blommaart EF, Luiken JJ, Blommaart PJ, Van Woerkom GM, Meijer AJ. Phosphorylation of ribosomal protein $\mathrm{S} 6$ is inhibitory for autophagy in isolated rat hepatocytes. $\mathrm{J} B i o l$ Chem 1995; 270: 2320-2326.

65. Klionsky DJ, Emr SD. Autophagy as a regulated pathway of cellular degradation [in process citation]. Science 2000; 290: 1717-1721.

66. Seglen PO, Gordon PB, Poli A. Amino acid inhibition of the autophagic/lysosomal pathway of protein degradation in isolated rat hepatocytes. Biochim Biophys Acta 1980; 630: $103-118$.

67. Van Sluijters DA, Dubbelhuis PF, Blommaart EF, Meijer AJ. Amino-acid-dependent signa ransduction [in process citation]. Biochem J 2000; 351 (Part 3): 545-550.

68. Eskelinen EL, Prescott AR, Cooper J, Brachmann SM, Wang L, Tang X et al. Inhibition of autophagy in mitotic animal cells. Traffic 2002; 3: 878-893.

69. Petiot A, Ogier-Denis E, Blommaart EF, Meijer AJ, Codogno P. Distinct classes of phosphatidylinositol $3^{\prime}$-kinases are involved in signaling pathways that control macroautophagy in HT-29 cells. J Biol Chem 2000; 275: 992-998.

70. Blommaart EF, Krause U, Schellens JP, Vreeling-Sindelarova $H$, Meijer AJ. The phosphatidylinositol 3-kinase inhibitors wortmannin and LY294002 inhibit autophagy in isolated rat hepatocytes. Eur J Biochem 1997; 243: 240-246.

71. Takano-Ohmuro $\mathrm{H}$, Mukaida M, Kominami E, Morioka K. Autophagy in embryonic erythroid cells: its role in maturation. Eur J Cell Biol 2000; 79: 759-764.

72. Suzuki K, Kubota Y, Sekito T, Ohsumi Y. Hierarchy of Atg proteins in preautophagosomal structure organization. Genes Cells 2007; 12: 209-218.

73. Suzuki K, Ohsumi Y. Molecular machinery of autophagosome formation in yeast, Saccharomyces cerevisiae. FEBS Lett 2007; 581: 2156-2161.

74. Kihara A, Noda T, Ishihara N, Ohsumi Y. Two distinct Vps34 phosphatidylinositol 3-kinase complexes function in autophagy and carboxypeptidase $Y$ sorting in Saccharomyces cerevisiae. J Cell Biol 2001; 152: 519-530.

75. Liang C, Sir D, Lee S, Ou JH, Jung JU. Beyond autophagy: the role of UVRAG in membrane trafficking. Autophagy 2008; 4: 817-820.

76. Gutierrez MG, Munafo DB, Beron W, Colombo MI. Rab7 is required for the normal progression of the autophagic pathway in mammalian cells. J Cell Sci 2004; 117: 2687-2697.

77. Jager S, Bucci C, Tanida I, Ueno T, Kominami E, Saftig P et al. Role for Rab7 in maturation of late autophagic vacuoles. J Cell Sci 2004; 117: 4837-4848

78. Ravikumar B, Imarisio S, Sarkar S, O'Kane CJ, Rubinsztein DC. Rab5 modulates aggregation and toxicity of mutant huntingtin through macroautophagy in cell and fly models of Huntington disease. J Cell Sci 2008; 121: 1649-1660.

79. Ding WX, Yin XM. Sorting, recognition and activation of the misfolded protein degradation pathways through macroautophagy and the proteasome. Autophagy 2008 4: $141-150$.

80. Hoyer-Hansen M, Jaattela M. AMP-activated protein kinase: a universal regulator of autophagy? Autophagy 2007; 3: 381-383
81. Gordon PB, Holen I, Fosse M, Rotnes JS, Seglen PO. Dependence of hepatocytic autophagy on intracellularly sequestered calcium. J Biol Chem 1993; 268: 26107-26112.

82. Hoyer-Hansen M, Bastholm L, Szyniarowski P, Campanella M, Szabadkai G, Farkas T et al. Control of macroautophagy by calcium, calmodulin-dependent kinase kinase-beta, and Bcl-2. Mol Cell 2007; 25: 193-205.

83. Hoyer-Hansen M, Jaattela M. Connecting endoplasmic reticulum stress to autophagy by unfolded protein response and calcium. Cell Death Differ 2007; 14: 1576-1582.

84. Savina A, Furlan M, Vidal M, Colombo MI. Exosome release is regulated by a calciumdependent mechanism in K562 cells. J Biol Chem 2003; 278: 20083-20090.

85. Savina A, Fader CM, Damiani MT, Colombo MI. Rab11 promotes docking and fusion of multivesicular bodies in a calcium-dependent manner. Traffic 2005; 6: 131-143.

86. Abeliovich H, Zhang C, Dunn Jr WA, Shokat KM, Klionsky DJ. Chemical genetic analysis of Apg1 reveals a non-kinase role in the induction of autophagy. Mol Biol Cell 2003; 14 477-490.

87. Fass E, Shvets E, Degani I, Hirschberg K, Elazar Z. Microtubules support production of starvation-induced autophagosomes but not their targeting and fusion with lysosomes. J Biol Chem 2006; 281: 36303-36316.

88. Kochl R, Hu XW, Chan EY, Tooze SA. Microtubules facilitate autophagosome formation and fusion of autophagosomes with endosomes. Traffic 2006; 7: 129-145.

89. Nara A, Mizushima N, Yamamoto A, Kabeya Y, Ohsumi Y, Yoshimori T. SKD1 AAA ATPase-dependent endosomal transport is involved in autolysosome formation. Cell Struct Funct 2002; 27: 29-37.

90. Fader $\mathrm{CM}$, Colombo MI. Multivesicular bodies and autophagy in erythrocyte maturation. Autophagy 2006; 2: 122-125.

91. Zeng $\mathrm{X}$, Overmeyer $\mathrm{JH}$, Maltese WA. Functional specificity of the mammalian Beclin-Vps34 PI 3-kinase complex in macroautophagy versus endocytosis and lysosomal enzyme trafficking. J Cell Sci 2006; 119: 259-270.

92. Iwata A, Riley BE, Johnston JA, Kopito RR. HDAC6 and microtubules are required for autophagic degradation of aggregated huntingtin. J Biol Chem 2005; 280: 40282-40292.

93. Iwata J, Ezaki J, Komatsu M, Yokota S, Ueno T, Tanida I et al. Excess peroxisomes are degraded by autophagic machinery in mammals. J Biol Chem 2006; 281: 4035-4041.

94. Ravikumar B, Rubinsztein DC. Can autophagy protect against neurodegeneration caused by aggregate-prone proteins? NeuroReport 2004; 15: 2443-2445

95. Yamamoto A, Cremona ML, Rothman JE. Autophagy-mediated clearance of huntingtin aggregates triggered by the insulin-signaling pathway. J Cell Biol 2006; 172: 719-731.

96. Parkinson N, Ince PG, Smith MO, Highley R, Skibinski G, Andersen PM et al. ALS phenotypes with mutations in CHMP2B (charged multivesicular body protein 2B). Neurology 2006; 67: 1074-1077.

97. Skibinski G, Parkinson NJ, Brown JM, Chakrabarti L, Lloyd SL, Hummerich $\mathrm{H}$ et al. Mutations in the endosomal ESCRTIII-complex subunit CHMP2B in frontotemporal dementia. Nat Genet 2005; 37: 806-808.

98. Lee JA, Beigneux A, Ahmad ST, Young SG, Gao FB. ESCRT-III dysfunction causes autophagosome accumulation and neurodegeneration. Curr Biol 2007; 17: 1561-1567.

99. Rusten TE, Filimonenko M, Rodahl LM, Stenmark H, Simonsen A. ESCRTing autophagic clearance of aggregating proteins. Autophagy 2007; 4: 1166-1172.

100. Philips JA, Porto MC, Wang H, Rubin EJ, Perrimon N. ESCRT factors restrict mycobacterial growth. Proc Natl Acad Sci USA 2008; 105: 3070-3075.

101. Rusten TE, Simonsen A. ESCRT functions in autophagy and associated disease. Cell Cycle 2008; 7: 1166-1172. 